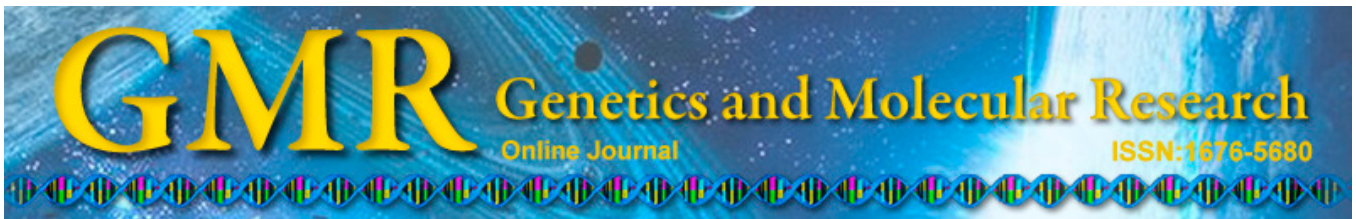

\title{
Aspergillus fumigatus diffusates suppress polymorphonuclear neutrophil phagocytic functions and respiratory burst levels in hematopoietic stem cell transplantation patients
}

\author{
X.H. Chen ${ }^{1}$, Y.C. Deng ${ }^{2}$, B.Y. Zhong ${ }^{1}$ and F. Hao ${ }^{1}$ \\ ${ }^{1}$ Department of Dermatology, Southwest Hospital, \\ Third Military Medical University, Chongqing, China \\ ${ }^{2}$ Department of Pharmacy, Southwest Hospital, \\ Third Military Medical University, Chongqing, China \\ Corresponding author: F. Hao \\ E-mail: FeiHao92001@163.com
}

Genet. Mol. Res. 14 (3): 9233-9243 (2015)

Received January 26, 2015

Accepted March 23, 2015

Published August 10, 2015

DOI http://dx.doi.org/10.4238/2015.August.10.3

\begin{abstract}
Invasive aspergillosis (IA) is a severe infection that commonly occurs in immunocompromised patients after hematopoietic stem cell transplantation (HSCT). The present study explores the effect of Aspergillus fumigatus diffusates (AfDs) on phagocytic function and superoxide anion $\left(\mathrm{O}_{2}^{-}\right)$burst levels in polymorphonuclear neutrophils (PMNs) from post-HSCT patients. A. fumigatus conidia with or without AfD were used to stimulate the PMN from healthy donor or HSCT patient for two hours. PMN morphology was visualized by scanning electron microscopy. The levels of respiratory burst $\mathrm{O}_{2}^{-}$produced by the PMNs were determined by flow cytometry. PMN phagocytic rates and phagocytic indexes were observed and calculated using periodic acid-Schiff (PAS) staining under a light-field microscope. No difference
\end{abstract}


was found between the PMN phagocytic rates, phagocytic indexes, or $\mathrm{O}_{2}^{-}$respiratory burst levels in health donor PMNs following treatments of $A$. fumigatus conidia with or without AfD. However, significant inhibition of these indices was seen in the PMNs from HSCT patients following treatment of A. fumigatus conidia plus $\mathrm{AfD}$, compared to that with conidium treatment alone $(\mathrm{P}<0.05)$. Therefore, AfD significantly inhibited the phagocytic function of PMNs from HSCT patients, potentially through inhibition of intracellular respiratory burst levels during phagocytosis. This suggests that the reason underlying the greater susceptibility of HSCT patients to aspergillosis might be the existence of AfD in vivo during infection. Further research on the mechanisms by which AfD affects the phagocytic function of PMNs from HSCT patients is therefore of great significance for the prevention of IA.

Key words: Hematopoietic stem cell transplantation; Superoxide anion; Invasive aspergillosis; Aspergillus fumigatus diffusates; Phagocytic rate; Polymorphonuclear neutrophils

\section{INTRODUCTION}

Aspergillus fumigatus is the most commonly occurred pathogen in invasive aspergillosis (IA) (Rivera et al., 2005). IA is a severe infection that commonly occurs in immunocompromised patients, which has few effective treatment choices, thus yielding a mortality rate as higher as $80 \%$ (Solé et al., 2005; Iversen et al., 2007). As a key component of the human innate immune system, polymorphonuclear neutrophils (PMNs) participate in the nonspecific anti-infection response during infection. However, the mechanism of A. fumigatus clearance by PMNs is not fully understood. Studies have found that remnant $A$. fumigatus can be transformed from conidia to mycelium form within PMNs, suggesting an important role for PMNs in mycelium removal. This model has been supported by other studies wherein cultured diffusates of $A$. fumigatus (AfDs) were shown to have immunosuppressive effects on PMNs (Higurashi et al., 2007). Previous studies have elucidated an indispensable role for PMNs in the clearance of $A$. fumigatus (Ikegami et al., 1998). It has been recognized that PMN respiratory bursts can produce superoxide anions $\left(\mathrm{O}_{2}^{-}\right)$to inactivate the iron-sulfur proteins in microbes and effectively kill invading pathogens or tumor cells, thus playing an important role in host defense and inflammatory response (Witko-Sarsat et al., 2000). In clinical practice, post-hematopoietic stem cell transplantation (HSCT) patients are susceptible to fungal infections at the early post-transplantation stage (Wang et al., 2008). However, the ability of and possible mechanisms utilized by PMNs in post-HSCT patients to clear A. fumigatus have not yet been reported.

In the present study, we explored the change in phagocytic function and respiratory burst levels of PMNs from post-HSCT patients during A. fumigatus infection in vitro. We hypothesized that AfD affected the phagocytic function of post-HSCT patient PMNs through repression of their respiratory burst levels during A. fumigatus infection. To test this hypothesis, we ascertained the phagocytic rates, phagocytic indexes, and $\mathrm{O}_{2}^{-}$respiratory burst levels of PMNs from both healthy donors and post-HSCT patients, through stimulation of their PMNs with A. fumigatus conidia with or without AfD. 


\section{MATERIAL AND METHODS}

\section{General information}

The Ethical Committee of the Third Military Medical University approved this study and confirmed that this research was in accordance with the Helsinki Declaration of 1975 (as revised in 1983). All patients $(\mathrm{N}=8)$ recruited in this study received HSCT within three weeks from the Department of Hematology at Southwest Hospital, and had normal white blood cell counts $\left(3-8 \times 10^{9} / \mathrm{L}\right)$. Healthy donors $(\mathrm{N}=8)$, selected from blood donors in our hospital with normal white blood cell counts $\left(4-8 \times 10^{9} / \mathrm{L}\right)$, were taken as controls. The general information of the patients and healthy controls is listed in Table 1.

Table 1. General information of the patients and controls.

\begin{tabular}{lcc}
\hline & Controls & Stem cell recipients \\
\hline Age (years) & $33.9 \pm 7.3$ & $29.3 \pm 7.4$ \\
Body weight $(\mathrm{kg})$ & $66.7 \pm 4.5$ & $61.8 \pm 5.9$ \\
No. of males (\%) & $4(50 \%)$ & $4(50 \%)$ \\
\hline
\end{tabular}

\section{Fungal strains, reagents, and equipment}

The A. fumigatus strain $\mathrm{CMCCC}(\mathrm{F}) \mathrm{A}_{1}$ a was kindly provided by the Nanjing Dermatology Institute (Nanjing, China). Human PMN separation buffer (Catalog No. LTS1087) was purchased from Jinmai Genetic Corp.(Tianjing, China). RPMI1640 and fetal bovine serum (FBS) were purchased from Hyclone Labs (Logan, UT, USA). The periodic acid-schiff staining (PAS) staining kit was purchased from Sigma (St Louis, MO, USA). Phosphate buffer solution (PBS) (0.15 M, pH 7.4) as well as a high-speed frozen centrifuge (Model ST40R) were purchased from Thermo Fisher Scientific (Waltham, MA, USA). The high-speed centrifuge (2-16) was a product of Sigma Corp. Giemsa and trypan blue dyes were prepared in-house by the Central Laboratory of Southwest Hospital. The reactive oxygen assay kit (DCFH-DA, Catalog No. S0021) was purchased from Beyotime Institute of Biotechnology (Shanghai, China). The carboxyfluorescein succinimydl ester (CFSE) test kit was provided by eBioscience (San Diego, CA, USA). The cell flow cytometer was produced by Becton Dickinson Corp. (Bedford, MA, USA). Other reagents used were locally produced at analytically pure grades.

\section{Preparation of the A. fumigatus strain and A. fumigatus diffusates (AfDs)}

The fungal strain was sub-cultivated onto freshly made Sandburg chloramphenicol agar culture medium. After incubation at $37^{\circ} \mathrm{C}$ for 4 days, the mycothallus was eluted using saline and vibrated with glass beads for $20 \mathrm{~min}$. The mixture was then filtered, diluted to make a conidia mixture at $1 \times 10^{7} \mathrm{CFU} / \mathrm{mL}$, and stored at $4^{\circ} \mathrm{C}$ for further use.

AfD was prepared as follows: The A. fumigatus conidia mixture was incubated in Hank's balance saline solution (HBSS) at $37^{\circ} \mathrm{C}$ for $1 \mathrm{~h}$, and filtered through a $0.22 \mu \mathrm{m}$ pinhole membrane filter. The flow-through solution was then centrifuged at $15,000 \mathrm{rpm}$ for $30 \mathrm{~min}$, and the pellets were resuspended with HBSS. These steps were repeated six times to get pure $\mathrm{AfD}$, which was stored at $-20^{\circ} \mathrm{C}$ for further use (Lopez et al., 1986). 


\section{Separation and purification of PMNs from peripheral blood}

Venous blood samples $(10 \mathrm{~mL}$; using heparin as an anticoagulant) from healthy donors or HSCT patients were mixed with equal volumes PBS buffer. An equal volume human neutrophils separation buffer was added and then centrifuged at $2000 \mathrm{rpm}$ for $20 \mathrm{~min}$ at room temperature; clear separated white layers above the serum could be observed following centrifugation. After an additional $2000 \mathrm{rpm}$ centrifugation for $10 \mathrm{~min}$, the white layers were gently resuspended using a micropipette filled with PBS buffer. The supernatants were collected and centrifuged at $2000 \mathrm{rpm}$ for $10 \mathrm{~min}$. The resultant supernatants were discarded, and the PMNs were resuspended in in PBS buffer. Living cells were quantified by the trypan blue exclusion method and $>98 \%$ living cells were identified in all samples collected (Figure 1). The PMNs were diluted in PBS buffer to generate $1 \times 10^{6} / \mathrm{mL}$ cell suspensions for future use (Li et al., 2008).

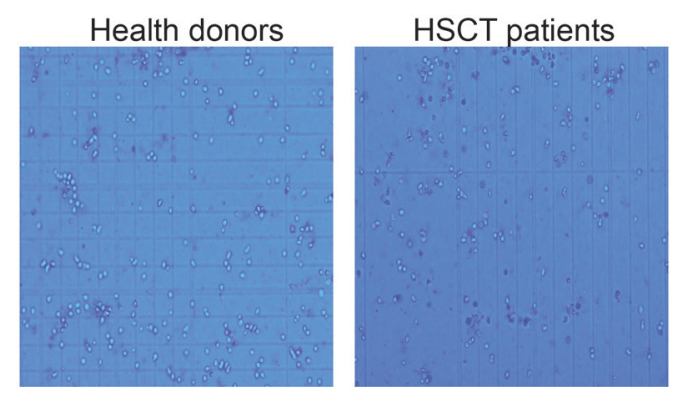

Figure 1. Analysis of PMN cell viability by trypan blue stain after separation and purification. After the separation of PMN, trypan blue staining was employed to observe the viability of PMN cells from healthy donors (left panel, 200X, $\mathrm{N}=10$ ) or post-HSCT patients (right panel, 200X, $\mathrm{N}=8$ ). Representative pictures from both group were shown.

\section{Giemsa staining and scanning electron microscopy of PMN cellular morphology}

Blood cell smear slides were generated from $0.2 \mathrm{~mL}$ PMN suspensions $\left(1 \times 10^{6} / \mathrm{mL}\right)$ of healthy donors and HSCT patients. Slides adhered with PMN were fixed in $95 \%$ ethanol for $10 \mathrm{~min}$, stained with several drops of Giemsa dye and buffer for $5 \mathrm{~min}$, washed with distilled water, dried, and observed under a light-field microscope.

For preparation of the samples for scanning electron microscopy, $0.5 \mathrm{~mL}$ PMN suspensions from each group were horizontally centrifuged at $2000 \mathrm{rpm}$ for $10 \mathrm{~min}$. After discarding the supernatant, cells were fixed in $3 \%$ glutaraldehyde solutions for $3 \mathrm{~h}$. Samples were sent to the Electron Microscopic Laboratory in the Third Military Medical University for embedding and sectioning.

\section{Determination of the level of PMN phagocytosis of $A$. fumigatus conidia by flow cytometry}

A $0.5 \mathrm{~mL}$ sample of $A$. fumigatus conidia mixture suspension was mixed with $2 \mathrm{~mL}$ PBS buffer to re-suspend the conidia, and then centrifuged at $8000 \mathrm{rpm}$ for $5 \mathrm{~min}$. The supernatant was discarded and the pellet re-suspended in $1 \mathrm{~mL}$ CFSE solution $(1 \mu \mathrm{M})$, followed by incubation at $37^{\circ} \mathrm{C}$ for $15 \mathrm{~min}$. The suspension was then washed 3 times with PBS buffer, resuspended in RPMI 1640 plus $10 \%$ FBS, and incubated with 2 x $10^{6}$ PMNs from healthy donors 
or HSCT patients at $37^{\circ} \mathrm{C}$ for $2 \mathrm{~h}$. After a gentle wash in PBS buffer, flow cytometry was used to measure the mean fluorescent intensities (MFIs) of the CFSE in PMN cells (Chitarra et al., 2005).

\section{Determination of the PMN phagocytic ratios and indexes by PAS staining}

Samples of PMN suspensions $\left(0.5 \mathrm{~mL} ; 1 \times 10^{6} / \mathrm{mL}\right)$ from healthy donors or HSCT patients were inoculated into separate 12 -well plates that with a coverslip in each well already, followed by addition of $10 \mu \mathrm{L}$ A. fumigatus conidia mixture with or without $10 \mu \mathrm{L}$ AfD. The total volume in each well was adjusted to $1 \mathrm{~mL}$ with PBS buffer. After $2 \mathrm{~h}$ incubation at $37^{\circ} \mathrm{C}$, coverslips from PMN-inoculated wells were taken for further PAS dye staining, according to the instructions provided by the company. The phagocytic rates and phagocytic indexes were calculated as follows: phagocytic rate $=100 \mathrm{x}$ the number of PMNs with inclusions of conidia/total cell number; phagocytic index $=100 \mathrm{x}$ the total number of phagocytic conidia/total cell number.

\section{Determination of $\mathrm{PMN}$ respiratory burst $\mathrm{O}_{2}^{-}$levels by flow cytometry}

Samples of PMN suspensions $\left(0.5 \mathrm{~mL} ; 1 \times 10^{6} / \mathrm{mL}\right)$ from healthy donors or HSCT patients were incubated with $10 \mu \mathrm{M}$ dichloro-dihydro-fluorescein diacetate (DCFH-DA) solution at $37^{\circ} \mathrm{C}$ for $15 \mathrm{~min}$. After being washed with HS buffer, the DCFH-DA loaded PMNs were co-cultured with $10 \mu \mathrm{L}$ A. fumigatus conidia suspensions with or without $10 \mu \mathrm{L}$ AfD in $1 \mathrm{~mL}$ total volume HS buffer. After $2 \mathrm{~h}$ incubation at $37^{\circ} \mathrm{C}$, the cells were washed with $\mathrm{HS}$ buffer three times followed by flow cytometric analysis. The DCF MFIs of the sample were taken to quantify the burst levels of $\mathrm{O}_{2}^{-}$in the PMNs (Imrich and Kobzik, 1998).

\section{Statistical analysis}

All data are reported as means \pm SD. SPSS ver. 10.0 (SPSS Corp., Chicago, IL, USA) was utilized for statistical analysis. One-way ANOVA was used for multiple comparisons. Statistical significance was defined as $\mathrm{P}<0.05$.

\section{RESULTS}

\section{Basic morphology of PMN cells}

Under Giemsa staining, PMNs from healthy donors displayed a rod shape or exhibited 2-5 lobes between which thin connecting filaments existed. In the PMNs from HSCT patients, the nuclear cytosol showed an enlargement, along with a higher proportion of myelocytes appeared as lobular eosinophils (Figure 2A). Flow cytometry analysis showed that the FSC and SSC values of the post-HSCT patient PMNs were significantly larger than those from healthy donor PMNs. These findings suggested a larger size and density of PMN cells in post-HSCT patients, compared with health donors (Figure 2B). By using scanning electron microscopy, we observed a relatively evenly distribution of cell sizes in healthy donor PMNs, whereas HSCT patients had PMNs of abnormally larger size and with uneven size distributions, with some cells remaining at an immature stage (Figure 2C). 

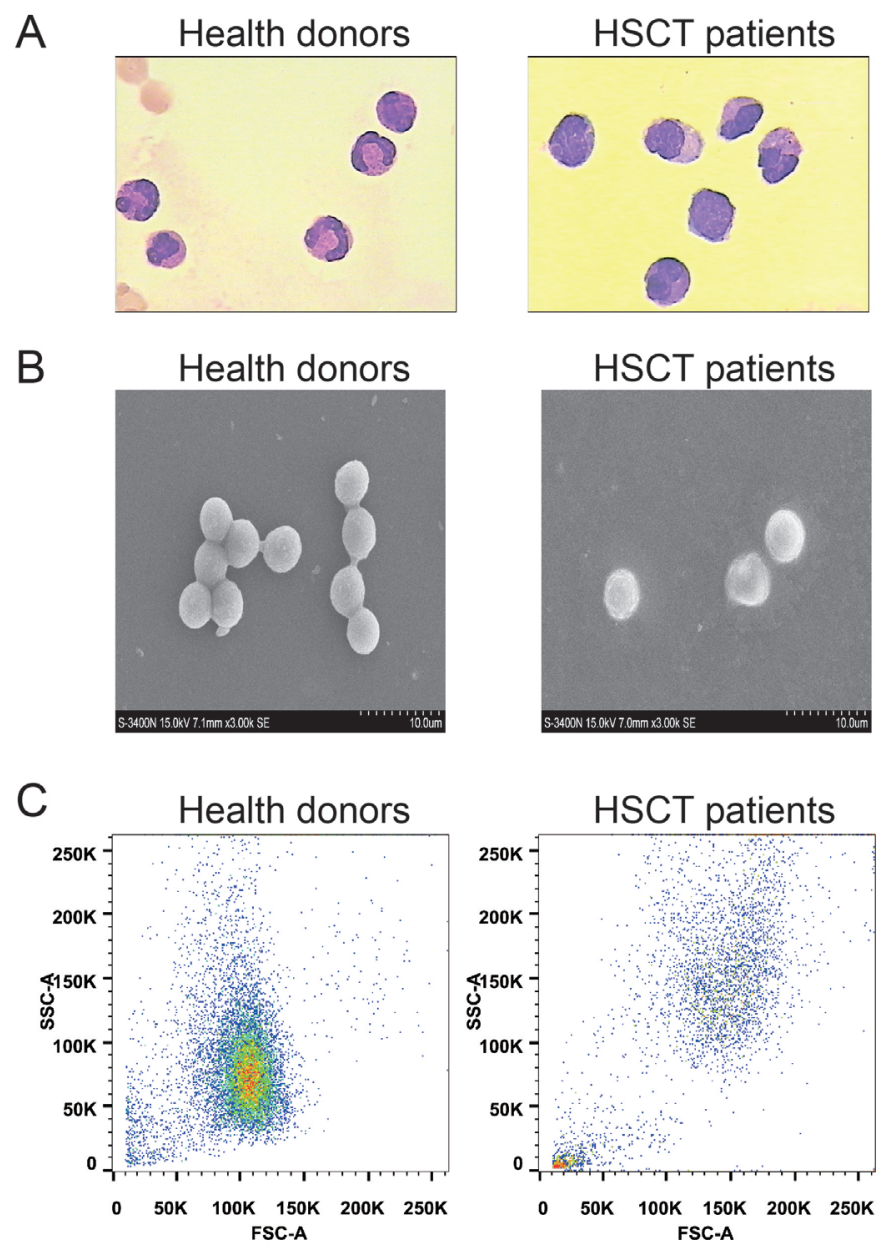

Figure 2. PMN cell morphology. A. Peripheral PMNs from healthy donors (left panel, $N=8$ ) and patients postHSCT (HSCT, right panel, $\mathrm{N}=4$ ) under Giemsa staining were observed for cellular size and nuclear morphology. B. Scanning electron microscopic observation of PMN cellular morphology in healthy donors $(\mathrm{N}=5)$ and patients post-HSCT $(\mathrm{N}=4)$. C. Distribution of the FSC and SSC of peripheral blood PMNs from healthy donors $(\mathrm{N}=6)$ and patients post-HSCT $(\mathrm{N}=5)$ using flow cytometry. PMNs, polymorphonuclear neutrophils; HSCT, hematopoietic stem cell transplantation; FSC, forward-scattered light; SSC, side-scattered light.

\section{Quantification of CFSE-positive conidia levels inside PMNs using flow cytometry}

After co-culture of PMNs with CFSE-labeled A. fumigatus conidia with or without AfD for $2 \mathrm{~h}$, flow cytometry was used to quantify PMN CFSE MFIs. The results showed no significant differences in CFSE MFIs between A. fumigatus conidia stimulation with or without AfD in PMN healthy donor (P > 0.05). However, in HSCT patients, the CFSE MFIs in PMNs were significantly decreased when conidia plus AfD were used for co-culture, compared with that with $A$. fumigatus conidia stimulation alone $(\mathrm{P}<0.05)$. These results suggested that there was an inhibitory effect on PMN phagocytosis of conidia resulting from AfD (Figure 3). 


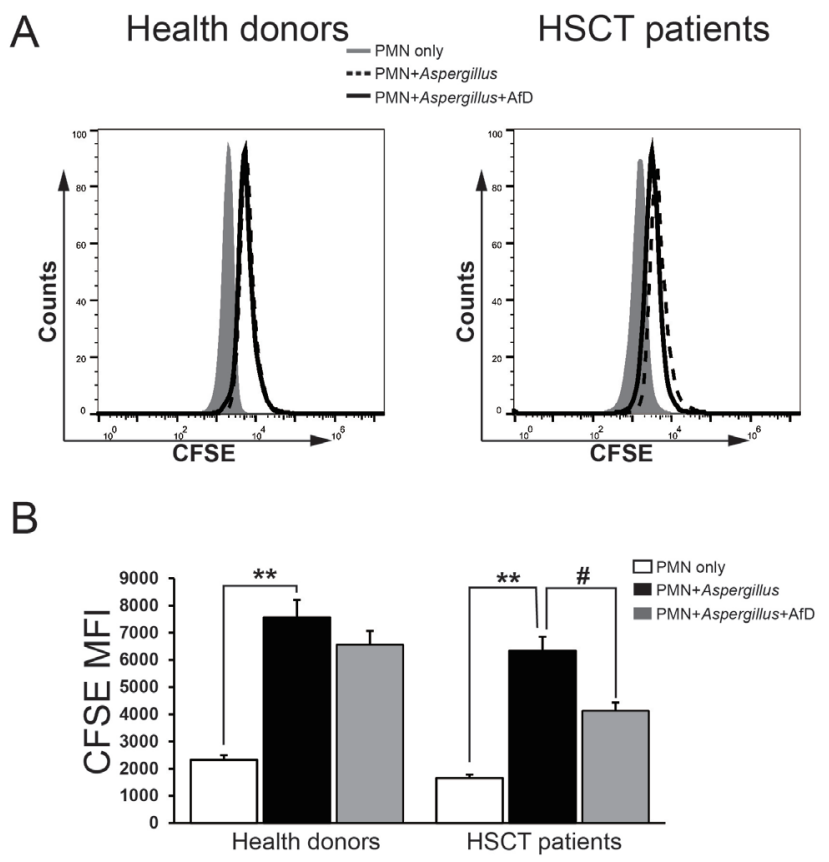

Figure 3. CFSE-positive conidia levels in different PMN samples by flow cytometry. After two hours co-incubation of peripheral separated PMNs with a CFSE-labeled conidia mixture or a conidia plus Afd mixture, flow cytometry was used to determine the mean fluorescent intensity (MFI) of CFSE in PMNs. A. Representative data from healthy donors (left panel) and post-HSCT patients (right panel). B. Statistical analysis of data from A. **P $<0.01$, compared to PMNs only; ${ }^{*} \mathrm{P}<0.05$, compared to PMNs plus Aspergillus. Error bars represent SD. $\mathrm{N}=6$ or 4 in healthy donors or patients post-HSCT, respectively. CFSE, carboxyfluorescein succinimidyl ester; Afd, Aspergillus fumigatus diffusates; PMN, polymorphonuclear neutrophil; HSCT, hematopoietic stem cell transplantation.

\section{Measurement of the phagocytic rates and indexes of PMNs by PAS staining}

To ascertain the phagocytic rates and indexes, PAS staining was performed after $2 \mathrm{~h}$ coculture of PMNs with A. fumigatus conidia suspensions with or without AfD. The results showed that there were no significant differences in the phagocytic rates or indexes between the conidia and conidia plus AfD treatments in PMN healthy donor $(\mathrm{P}>0.05)$. However, we found that AfD significantly inhibited the phagocytic rates and indexes in HSCT patient PMNs, compared with those obtained using co-culture with $A$. fumigatus conidia alone $(\mathrm{P}<0.05$; Figure 4$)$.

\section{Flow cytometric analysis of $\mathrm{PMN}$ respiratory burst $\mathrm{O}_{2}^{-}$levels}

To determine the PMN respiratory burst $\mathrm{O}_{2}^{-}$levels, we detected the intracellular DCF MFIs, indicators of intracellular $\mathrm{O}_{2}^{-}$, by flow cytometry after $2 \mathrm{~h}$ co-culture of PMNs with A. fumigatus conidia with or without AfD. Results showed that there were no significant differences of $\mathrm{O}_{2}^{-}$burst levels in healthy donor PMNs following stimulation by conidia with or without AfD $(\mathrm{P}>0.05)$. However, in HSCT patients, the $\mathrm{O}_{2}^{-}$burst levels of PMNs after stimulation by conidia plus AfD were significantly lower than those in cells stimulated by conidia alone $(\mathrm{P}<0.05)$ (Figure 5). 
A
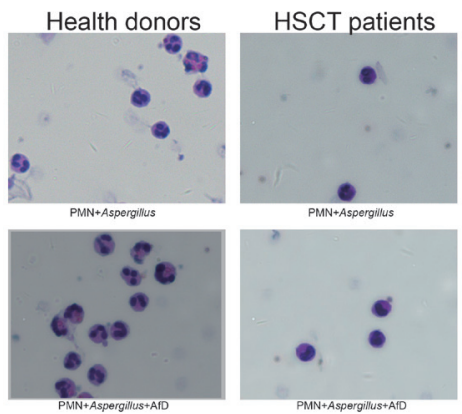

B
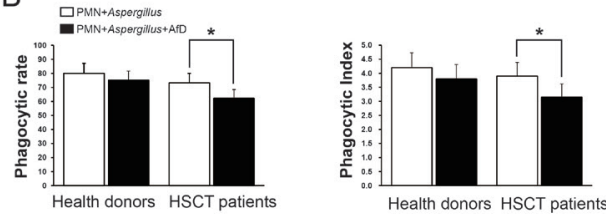

Figure 4. PMN phagocytic rates and indexes of Aspergillus fumigatus conidia. After $2 \mathrm{~h}$ co-incubation of PMNs with a conidia mixture or a conidia + AfD suspension, PAS staining was used to measure the phagocytic rates and indexes of PMN phagocytosis of Aspergillus fumigatus conidia. A. Representative pictures from healthy donors (left panel) and patients post-HSCT (right panel). B. Statistical analysis of data from A. ${ }^{*} \mathrm{P}<0.05$, compared to PMNs plus Aspergillus. Error bars represent $\mathrm{SD}(\mathrm{N}=6$ or 4 in healthy donors or patients post-HSCT, respectively). PMNs, polymorphonuclear neutrophils; Afd, Aspergillus fumigatus diffusates; PAS, periodic acid-Schiff staining.

A

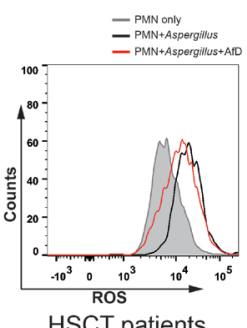

B

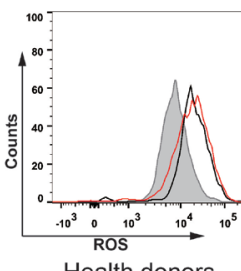

HSCT patients

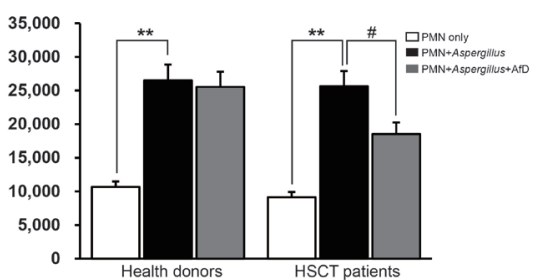

Figure 5. Flow cytometric measurement of PMN respiratory burst $\mathrm{O}_{2}^{-}$levels. After co-incubation with a conidia mixture suspension or a conidia plus AfD mixture for $2 \mathrm{~h}$, the peripheral separated $\mathrm{PMN} \mathrm{O}_{2}^{-}$burst levels (ROS) were quantified by flow cytometry. A. Representative data from healthy donors (left panel) and patients post-HSCT (right panel) are shown. B. Statistical data from A. ${ }^{* *} \mathrm{P}<0.01$, compared to PMNs only; ${ }^{*} \mathrm{P}<0.05$ compared to $\mathrm{PMN}+$ Aspergillus. Error bars represent SD $(\mathrm{N}=6$ or 4 in healthy donors or patients post-HSCT, respectively). PMN, polymorphonuclear neutrophils; Afd, Aspergillus fumigatus diffusates. 


\section{DISCUSSION}

HSCT by cytokine-induced mobilization has been widely used as an effective therapy against various malignant hematological diseases (Gratwohl et al., 2001). Due to its multiple advantages over bone marrow transplantation (BMT), including a shorter recovery time for granulocytes, platelets, and hematopoietic functions, HSCT considerably shortens hospital stays and lowers transplantation expenses. These advantages resulted in peripheral blood rather than bone marrow becoming the standard source for allogeneic HSCT (Champlin et al., 2000; Couban et al., 2002). However, post-transplantation infection often occurs in HSCT patients, especially in those with immune deficiencies, due to their malnutrition and compromised immune functions caused by high dosage chemotherapy (Wu and Li, 2009). IA has an occurrence rate of $8-15 \%$ in HSCT recipients, and is observed most often in patients within three weeks following transplantation. The course of IA proceeds rapidly and causes death in about 1 or 2 weeks in some cases. Clinical statistics show an IA mortality rate as high as 30$80 \%$, even up to $100 \%$ mortality if without any effective treatment (Bellocchio et al., 2005). Therefore, infection by Aspergillus, albeit clinically uncommon, renders the prognosis of HSCT patients more unfavorable. In order to understand the ability of patient PMNs to defend against Aspergillus infection, it is useful to study the morphology of PMNs from post-HSCT patients. This study found that patient PMN cells appeared to be at a relatively immature stage three weeks after HSCT. Giemsa staining, scanning electron microscope and flow cytometric analysis also demonstrated a lower phagocytic ability of patient PMNs. Therefore, we validated the previously described correlation between PMN morphology and phagocytic ability in post-HSCT patients.

PMNs are an important component of the human immune system and are closely related to the nonspecific anti-infection process. PMNs are effective in the phagocytosis of pathogens, which involves a process including the chemotrophic attraction, recognition, binding, phagocytosis, inclusion, inactivation, and degradation of pathogens (Shurtz-Swirski et al., 2001). Multiple metabolites of A. fumigatus have been shown to interfere with cellular phagocytic functions (Hao, 2004). The conidia and mycelium of $A$. fumigatus can induce PMNs and other inflammatory cells to produce various cytokines such as tumor necrosis factor- $\alpha$ and interleukins- 1 and -6 , all of which play important roles in the clearance of A. fumigatus (Andrews and Sullivan, 2003). This study used flow cytometry and PAS staining to demonstrate that AfD significantly inhibited the phagocytic ability of PMNs toward conidia in HSCT patients but not in healthy donors as shown by the lower PMN phagocytic rates and indexes, thereby suggesting that AfD contributes to the high infection rate of A. fumigatus in early post-HSCT patients.

Respiratory bursts are a feature of PMNs in which reactive oxygen species $\left(\mathrm{O}_{2}^{-}\right)$are abundantly released during the phagocytosis of foreign materials. These free oxygen radicals play an indispensable role in killing invading microbes, protecting the body, and maintaining the homeostatic status (Zhao et al., 1989a,b). In other studies, IA was found to occur most commonly in secondary invasive fungal post-myelosuppression infections (Roden et al., 2005). This study found that the respiratory burst $\mathrm{O}_{2}^{-}$levels of PMNs from HSCT patients after a single stimulus of $A$. fumigatus conidia were not significantly different compared to those of PMNs from normal individuals. However, the respiratory burst $\mathrm{O}_{2}^{-}$levels of patient PMNs were significantly suppressed by the stimulus of conidia plus AfD. Furthermore, the respiratory burst $\mathrm{O}_{2}^{-}$level was positively correlated with the effect of AfD on the phagocytic function 
of HSCT patient PMNs. These results suggest that AfD probably affect the phagocytic ability of HSCT patient PMNs via inhibition of the PMN respiratory burst levels.

In conclusion, building upon the clinical observation that immunosuppressant patients are susceptible to A. fumigatus infections (Williamson et al., 2000; Loeffler et al., 2002; Petraitiene et al., 2002; Su et al., 2006), this study demonstrated that PMNs from HSCT patients exhibited immature morphology and impaired phagocytic functions, and that their phagocytic function could be significantly inhibited by AfD. This study provides the basis for further analysis of the effects of $A$. fumigatus conidia and their metabolites on PMN-mediated immune functions and related signaling pathways. This knowledge might lead to a new strategy for the prevention of nosocomial Aspergillus infections and the development of a bio-therapy or immune therapy against the pathogenic factors, which together will be the focus for our future research.

\section{Conflicts of interest}

The authors declare no conflict of interest.

\section{ACKNOWLEDGMENTS}

Research supported by the Natural Science Foundation of China (\#30671886 to F. Hao).

\section{REFERENCES}

Andrews T and Sullivan KE (2003). Infections in patients with inherited defects in phagocytic function. Clin. Microbiol. Rev. 16: 597-621.

Bellocchio S, Bozza S, Montagnoli C, Perruccio K, et al. (2005). Immunity to Aspergillus fumigatus: the basis for immunotherapy and vaccination. Med. Mycol. 43: S181-S188.

Champlin RE, Schmitz N, Horowitz MM, Chapuis B, et al. (2000). Blood stem cells compared with bone marrow as a source of hematopoietic cells for allogeneic transplantation. IBMTR Histocompatibility and Stem Cell Sources Working Committee and the European Group for Blood and Marrow Transplantation (EBMT). Blood 95: 3702-3709.

Chitarra GS, Breeuwer P, Rombouts FM, Abee T, et al. (2005). Differentiation inside multicelled macroconidia of Fusarium culmorum during early germination. Fungal Genet. Biol. 42: 694-703.

Couban S, Simpson DR, Barnett MJ, Bredeson C, et al. (2002). A randomized multicenter comparison of bone marrow and peripheral blood in recipients of matched sibling allogeneic transplants for myeloid malignancies. Blood 100: 1525-1531.

Gratwohl A, Passweg J, Baldomero H, Urbano-Ispizua A, et al. (2001). Hematopoietic stem cell transplantation activity in Europe 1999. Bone Marrow Transplant. 27: 899-916.

Hao F (2004). The research progress in causative agents and mechanism of action of Aspergillus fumigatus. Chin. J. Nosocomiol. 14: 119-120.

Higurashi H, Arai M, Watanabe A, Igari H, et al. (2007). Gene expression profiling of polymorphonuclear leukocytes treated with the culture filtrate of Aspergillus fumigatus and gliotoxin. Microbiol. Immunol. 51: 407-419.

Ikegami Y, Amitani R, Murayama T, Nawada R, et al. (1998). Effects of alkaline protease or restrictocin deficient mutants of Aspergillus fumigatus on human polymorphonuclear leukocytes. Eur. Respir. J. 12: 607-611.

Imrich A and Kobzik L (1998). Flow cytometric analysis of macrophage oxidative metabolism using DCFH. Methods Mol. Biol. 91: 97-108.

Iversen M, Burton CM, Vand S, Skovfoged L, et al. (2007). Aspergillus infection in lung transplant patients: incidence and prognosis. Eur. J. Clin. Microbiol. Infect. Dis. 26: 879-886.

Li JF, Liu WL, Shi XJ, Liu W, et al. (2008). Comparison of 4 popular methods for neutrophil isolation from human peripheral blood. Int. J. Pathol. Clin. Med. 28: 277-281.

Loeffler J, Kloepfer K, Hebart H, Najvar L, et al. (2002). Polymerase chain reaction detection of Aspergillus DNA in experimental models of invasive aspergillosis. J. Infect. Dis. 185: 1203-1206. 
Lopez AF, Williamson DJ, Gamble JR, Begley CJ, et al. (1986). Recombinant human granulocyte-macrophage colonystimulating factor stimulates in vitro mature human neutrophil and eosinophil function, surface receptor expression, and survival. J. Clin. Invest. 78: 1220-1228.

Petraitiene R, Petraitis V, Groll AH, Sein T, et al. (2002). Antifungal efficacy of caspofungin (MK-0991) in experimental pulmonary aspergillosis in persistently neutropenic rabbits: pharmacokinetics, drug disposition, and relationship to galactomannan antigenemia. Antimicrob. Agents Chemother. 46: 12-23.

Rivera A, Van Epps HL, Hohl TM, Rizzuto G, et al. (2005). Distinct CD4+-T-cell responses to live and heat-inactivated Aspergillus fumigatus conidia. Infect. Immun. 73: 7170-7179.

Roden MM, Zaoutis TE, Buchanan WL, Knudsen TA, et al. (2005). Epidemiology and outcome of zygomycosis: a review of 929 reported cases. Clin. Infect. Dis. 41: 634-653.

Shurtz-Swirski R, Sela S, Herskovits AT, Shasha SM, et al. (2001). Involvement of peripheral polymorphonuclear leukocytes in oxidative stress and inflammation in type 2 diabetic patients. Diabetes Care 24: 104-110.

Solé A, Morant P, Salavert M, Permán J, et al. (2005). Aspergillus infections in lung transplant recipients: risk factors and outcome. Clin. Microbiol. Infect. 11: 359-365.

Su MQ, Yue QH, Yang L, Fan X, et al. (2006). Mechanism of Aspergillus infection during hypoimmunity: an experimental research. Chin. J. Nosocomiol. 16: 738-741.

Wang ZY, Jiang EL, Zhang P, Wang H, et al. (2008). Invasive fungal infections after allogeneic hematopoietic stem cell transplantation and related risk factors. Zhongguo Shi Yan Xue Ye Xue Za Zhi 16: 618-622.

Williamson EC, Leeming JP, Palmer HM, Steward CG, et al. (2000). Diagnosis of invasive aspergillosis in bone marrow transplant recipients by polymerase chain reaction. Br. J. Haematol. 108: 132-139.

Witko-Sarsat V, Rieu P, Descamps-Latscha B, Lesavre P, et al. (2000). Neutrophils: molecules, functions and pathophysiological aspects. Lab. Invest. 80: 617-653.

Wu D and Li FQ (2009). Roles of macrophages in resisting Aspergillus fumigatus infection. J. Med. Postgraduates 22: 1206-1210.

Zhao BL, Li XJ, He RG, Jia WY, et al. (1989a). ESR studies on oxygen consumption during the respiratory burst of human polymorphonuclear leukocytes. Cell Biol. Int. Rep. 13: 317-323.

Zhao BL, Li XJ and Xin WJ (1989b). ESR studies on active oxygen radicals produced in the respiratory burst of human polymorphonuclear leukocytes. Cell Biol. Int. Rep. 13: 529-536. 